\title{
Theoretical Study on the Operation of the EU/KIT TE34,19-Mode Coaxial-Cavity Gyrotron at 170/204/238 GHz
}

\author{
Tobias Ruess ${ }^{1, *}$, Konstantinos Avramidis ${ }^{1}$, Gerd Gantenbein ${ }^{1}$, Stefan Illy $^{1}$, Zisis Ioannidis ${ }^{1}$, Jianbo Jin ${ }^{1}$, Parth Kalaria $^{1}$, \\ Ioannis Gr. Pagonakis ${ }^{1}$, Sebastian Ruess ${ }^{1,2}$, Tomasz Rzesnicki ${ }^{1}$, Manfred Thumm ${ }^{1,2}$, and John Jelonnek ${ }^{1,2}$
}

${ }^{1} \mathrm{IHM},{ }^{2} \mathrm{IHE}$, Karlsruhe Institute of Technology (KIT), Kaiserstr. 12, 76131 Karlsruhe, Germany

\begin{abstract}
The $170 \mathrm{GHz} 2 \mathrm{MW} \mathrm{TE}_{34,19}$-mode coaxial-cavity modular short-pulse pre-prototype gyrotron at KIT was recently modified in order to verify the multi-megawatt coaxial-cavity technology at longer pulses. In parallel, theoretical investigations on a possibility to operate the $170 \mathrm{GHz} \mathrm{TE}_{34,19}$-mode coaxial-cavity prototype at multiple frequencies up to $238 \mathrm{GHz}$ have been started, with a goal to find a configuration at which the tube could operate in the KIT FULGOR gyrotron test facility using the new $10.5 \mathrm{~T}$ SC magnet. This paper indicates which adjustments have to be made and show the feasibility of the multi-frequency operation. Small modifications at the gyrotron cavity will support an RF output power of more than $2 \mathrm{MW}$ at 170/204 GHz. Furthermore, a new gyrotron launcher has been designed capable of producing a Gaussian microwave beam with a Gaussian mode content of more than $96 \%$ at these frequencies.
\end{abstract}

\section{Introduction}

Gyrotrons are RF microwave sources used for fusion applications. These vacuum tubes are the sources for Electron Cyclotron Resonance Heating and Current Drive (ECRH\&CD) for plasma heating and stabilization in fusion devices. For example, at the stellarator W7-X in Greifswald, Germany, ten gyrotrons operating at $140 \mathrm{GHz}$ and at $1 \mathrm{MW}$ (1800s) RF output power have been tested and demonstrated an excellent reliable performance [1]. The experimental reactor ITER, under construction in Caderache, France will use a $24 \mathrm{MW}$ ECRH installation with $24170 \mathrm{GHz}, 1 \mathrm{MW}$ gyrotrons [2]. In a next step, electricity will be produced by the subsequent project called DEMO (Demonstration Power Plant) [3]. Fusion gyrotrons for DEMO and future FPP (Fusion Power Plants) will require multi-megawatt continuous-wave (CW) operation at multiple possible frequencies starting from $170 \mathrm{GHz}$ up to significantly above $200 \mathrm{GHz}$. The EU DEMO, planned as next step after ITER, is under development within the Horizon 2020 EUROfusion programme [3].

The EU ITER gyrotron is designed with the conventional hollow-cavity technology [4]. This technology shows quite a good performance and a reliable operation whereby studies at frequencies above $200 \mathrm{GHz}$ has been made [5]. However, there are two main issues regarding hollow-cavity gyrotrons: (i) increasing mode competition with raising mode order, which can be a problem at the operation above $200 \mathrm{GHz}$ and (ii) high voltage depression caused by space charge effect of the electron beam. To overcoming these issues, a gyrotron using a coaxial insert (coaxial-cavity gyrotron) can be used. The coaxial cavity reduces mode competition and voltage depression [6,7]. The first KIT modular coaxial-cavity pre-prototype gyrotron reached an RF output power of more than $2 \mathrm{MW}$ at $170 \mathrm{GHz}$ in short-pulse operation [8,9]. The operating design parameters are summarized in Tab. 1. Pushing forward this technology, the pre-prototype has been upgraded towards longer-pulse operation of $100 \mathrm{~ms}$, and towards $1 \mathrm{~s}$ in a second step [10]. For future DEMO gyrotrons, multi-frequency operation at e.g. $136 \mathrm{GHz}, 170 \mathrm{GHz}$, $204 \mathrm{GHz}$, or $238 \mathrm{GHz}$ would be highly beneficial. The aim of this work is to investigate if the operation at these frequencies is possible with the existing coaxial-cavity pre-prototype, using the KIT FULGOR gyrotron test facility [11] and a new $10.5 \mathrm{~T}$ Super-Conducting magnet, without a major effort on developing new components.

This paper is structured in the following way: Firstly, an overview about the mode selection for multifrequency operation is presented in section 2. In the

Table 1. Design Parameters for the Coaxial-Cavity Gyrotron [8]

\begin{tabular}{|l|c|}
\hline Operating mode & $\mathrm{TE}_{34,19}$ \\
\hline Frequency, $f$ & $170 \mathrm{GHz}$ \\
\hline RF output power, $P_{\text {out }}$ & $2 \mathrm{MW}$ \\
\hline Beam current, $I_{B}$ & $75 \mathrm{~A}$ \\
\hline Accelerating voltage, $U_{C}$ & $90 \mathrm{kV}$ \\
\hline Velocity ratio (pitch factor), $\alpha$ & $\sim 1.3$ \\
\hline Magnetic field at cavity, $B_{\text {cav }}$ & $6.87 \mathrm{~T}$ \\
\hline Outer cavity radius, $R_{\text {cav }}$ & $29.55 \mathrm{~mm}$ \\
\hline
\end{tabular}

\footnotetext{
* Corresponding author: tobias.ruess@ $@$ kit.edu
} 
following Section 3, initial studies regarding multifrequency operation using the magnetic field profile of an existing magnet are reported. Further, a study about the geometrical issues regarding the new configuration of the new 10.5 T SC magnet from Tesla Engineering Ltd., Storrington, UK is started in section 4. Taken this into account, realistic start-up scenarios are simulated under the consideration of the proposal for the coil configuration from Tesla Engineering Ltd. and real electron gun parameters, shown in section 5 . Comparisons are made between simulations addressing only the gyrotron cavity and simulations addressing both the cavity and the non-linear uptaper after the cavity. Additionally, a modification of the coaxial-cavity is proposed to increase the performance. In the last section the behaviour of the existing launcher is investigated and an advanced design is proposed showing promising results.

\section{Mode Selection for Multi-Frequency Operation}

The investigations are based on the existing coaxialcavity of the KIT pre-prototype. This cavity has a total length of $68 \mathrm{~mm}$ and a midsection length of $16 \mathrm{~mm}$. This corresponds to $\sim 9 \cdot \lambda_{170 \mathrm{GHz}}$ where $\lambda_{170 \mathrm{GHz}}$ denotes the freespace wavelength at $170 \mathrm{GHz}$. The possible previously mentioned operating frequencies should correspond to the natural $\lambda / 2$ resonances of the synthetic diamond output window using: $f_{\text {op }}=\left(N \cdot c_{0}\right) /\left(2 \cdot d_{\text {window }} \cdot \varepsilon_{r}{ }^{l / 2}\right)$ with $N \in \mathbb{N}$, where $c_{0}$ is the speed of light in vacuum, $d_{\text {window }}$ is the thickness of the window disk and $\varepsilon_{r}$ is the relative dielectric permittivity of the window material $\left(\varepsilon_{r}=5.67\right.$ for the existing Chemical Vapor Deposition diamond window). Window matching is the first condition for multi-frequency operation. The second condition is given by the launcher performance. Possible modes for multi-frequency operation should exhibit a maximum deviation of the caustic radius $R_{C}$ of $<3-4 \%$, where the caustic radius is determined by $R_{C}=m \cdot R_{o} /$ $\chi_{m, n}$. Here $R_{o}$ is the cavity radius and $\chi_{m, n}$ the mode eigenvalue, which is the $n^{\text {th }}$ root of the characteristic equation in the coaxial cavity [6]. This condition enables the usage of the existing, or at least of a similar, quasioptical system. The modes obtained, taking into account the mentioned considerations, are the $\mathrm{TE}_{40,23}$ mode operating at $204 \mathrm{GHz}$ and the modes $\mathrm{TE}_{48,26}$ and $\mathrm{TE}_{46,27}$ operating at $237 \mathrm{GHz}$ and $238 \mathrm{GHz}$, respectively. Table 2 summarizes the properties in terms of window

Table 2. Mode Selection for Multi-Frequency Operation.

\begin{tabular}{|l|c|c|c|c|}
\hline Mode & $\mathrm{TE}_{34,19}$ & $\mathrm{TE}_{40,23}$ & $\mathrm{TE}_{48,26}$ & $\mathrm{TE}_{46,27}$ \\
\hline $\begin{array}{l}\text { Frequency } \\
\text { [GHz] }\end{array}$ & 170 & 204.179 & 237.242 & 238.351 \\
\hline $\begin{array}{l}\text { Window } \\
\text { reflection [\%] }\end{array}$ & 0 & 0.026 & 0.472 & 0.101 \\
\hline $\begin{array}{l}\text { Rel. caustic } \\
\text { radius } R_{C} / R_{o}\end{array}$ & 0.3232 & 0.3167 & 0.3270 & 0.3119 \\
\hline $\begin{array}{l}\text { Diff. in caustic } \\
\text { radius [\%] }\end{array}$ & 0 & 2.01 & 1.18 & 3.62 \\
\hline
\end{tabular}

reflection and caustic radius of the chosen modes. The $\mathrm{TE}_{40,23}$ mode $\left(\chi_{40,23}=126.3\right)$ has a frequency close to the natural resonance frequency of the diamond window, which results in a very low window reflection. But the difference in caustic radius with respect to the $\mathrm{TE}_{34,19}$ mode is $2.01 \%$ and it has to be checked if the operation of the existing launcher and mirror system is still acceptable for this mode. In future, a compromise of the window thickness can be considered to improve the reflection coefficient at $204 \mathrm{GHz}$. The operation at $\sim 240 \mathrm{GHz}$ can be realised with two possible modes. One of them $\left(\mathrm{TE}_{48,26}, \chi=146.8\right)$ exhibits a relatively high window reflection, whereas the other $\left(\mathrm{TE}_{46,27,} \chi=147.5\right)$ has a high difference of the caustic radius. The simulation results, in terms of beam-wave interaction, are quite similar for these two modes. This means that the mode selection for operation at $\sim 240 \mathrm{GHz}$ should be made after calculating the behaviour of the launcher and mirror system. In the following, results with respect to beam-wave interaction are reported only for the mode $\mathrm{TE}_{48,26}$, for brevity.

\section{Simulations using the existing Oxford Instruments magnetic field profile}

In initial studies, the KIT pre-prototype cavity without the non-linear uptaper was considered and already presented in [12]. However, the simulation results will be repeated within this paper for completeness. There, the magnetic field profile of the existing $7 \mathrm{~T}$ Oxford Instruments superconductive (SC) magnet was used as a typical profile. It should be mentioned, however, that the existing magnet cannot deliver the needed magnetic field strength for frequencies above $170 \mathrm{GHz}$. The appropriate super-conducting magnet is under procurement and is expected to be delivered by mid-2019 by Tesla Engineering Ltd. (Cf. Section 5).

The beam-wave interaction in the cavity for operation at $170 / 204 / 238 \mathrm{GHz}$ was simulated using EURIDICE [13,14]. In order to be as realistic as possible at this initial stage of investigations, the simulations were done assuming a kinetic energy spread of $\Delta E=0.2 \% \mathrm{rms}$ and a relatively large spread of $\Delta \alpha=20 \% \mathrm{rms}$ for the electron velocity ratio $\alpha$. The adiabatic start-up scenario was simulated for this setup at $204 \mathrm{GHz}$ and $238 \mathrm{GHz}$ by linearly raising the accelerating voltage. By adiabatic one understands, that the complete electron trajectory from the emitter to the cavity is assumed adiabatic. The most important competing modes at $204 \mathrm{GHz}$ are shown in Fig. 1. The adiabatic start-up scenario simulation including 72 competing modes and a magnetic field of $8.15 \mathrm{~T}$ is shown in Fig. 2.

In the simulations, a lower velocity ratio $\alpha=1.2$ instead of $\alpha=1.3$ was used to reduce the risk of unwanted parasitic oscillations, especially at frequencies above $200 \mathrm{GHz}$. The simulation showed a good scenario starting with the $\mathrm{TE}_{41,23}$ mode. At $75.5 \mathrm{keV}$ the nominal $\mathrm{TE}_{40,23}$ mode becomes dominant. In this case the operating point is defined by the Ohmic loading constraints. These constraints limit the insert wall 
loading to $0.2 \mathrm{~kW} / \mathrm{cm}^{2}$ and the cavity wall loading to $2 \mathrm{~kW} / \mathrm{cm}^{2}$. The maximum acceptable cavity loading defines the operating parameters of $U_{\text {beam }}=80.7 \mathrm{keV}$ for the electron beam energy and $I_{\text {beam }}=70 \mathrm{~A}$ for the beam current. An RF output power of $1.8 \mathrm{MW}$ radiated from the cavity at an interaction efficiency of $33 \%$ is obtained at $204.14 \mathrm{GHz}$. The insert wall loading amounts to $0.065 \mathrm{~kW} / \mathrm{cm}^{2}$ and is far below the limit.

In order to improve the performance at the higher frequencies, the midsection length of the coaxial cavity was reduced to $\sim 9 \lambda_{204 \mathrm{GHz}}(13.6 \mathrm{~mm})$ as a first proposed modification. Reducing the midsection length leads to lower quality factor and thus the wall loading is decreased as well. Hence, the operating parameters of the beam voltage, beam current, and magnetic field can be further increased without violating the maximum allowable wall loading. As a result, the RF output power was also increased. In this case, the operation parameters for the operation at $204 \mathrm{GHz}$ are determined to be $U_{\text {beam }}=88 \mathrm{keV}, I_{\text {beam }}=75 \mathrm{~A}$, and $B=8.23 \mathrm{~T}$. The cavity $\mathrm{RF}$ output power was increased to $2.2 \mathrm{MW}$ with an interaction efficiency of $33.4 \%$.

At the next higher frequency $(\sim 238 \mathrm{GHz})$ the number of possible competing modes is increased to 90 . This effect comes from stronger mode competition due to a denser mode spectrum by raising the frequency. At $\sim 238 \mathrm{GHz}$ mode stability has been a problem, especially at high beam voltages and/or beam currents. The operation parameters have been reduced to $U_{\text {beam }}=60 \mathrm{keV}$ and $I_{\text {beam }}=60 \mathrm{~A}$. Therefore, the RF output power with the $\mathrm{TE}_{48,26}$ mode drops to $1.04 \mathrm{MW}$ at an efficiency of $30.2 \%$. One of the issues operating at higher frequencies with the same cavity is the comparatively long cavity, where electron overbunching can occur and so, the electron beam regains energy from the RF beam. To overcome this issue, the simulation was performed with the shorter midsection length of the coaxial cavity plus a reduced pitch factor $(\alpha=1.1)$ which leads to stable operation at $U_{\text {beam }}=78 \mathrm{keV}$, $I_{\text {beam }}=70 \mathrm{keV}$, and $B=9.45 \mathrm{~T}$. The cavity wall loading is $1.85 \mathrm{~kW} / \mathrm{cm}^{2}$ at that point. The output power reaches 1.6 MW with an efficiency of $30.4 \%$.

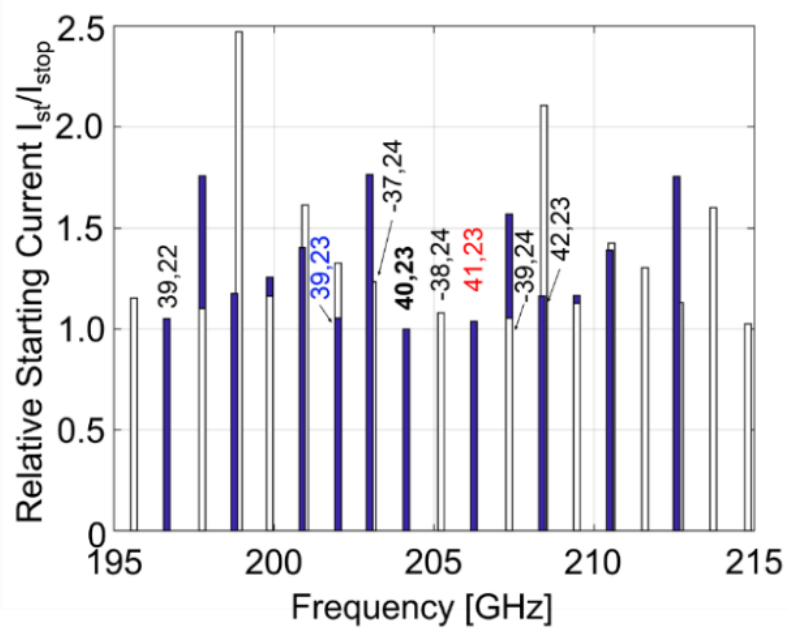

Fig. 1. Relative starting current of competing modes at $8.15 \mathrm{~T}$ and $I_{\text {beam }}=70$ A (filled bars: co-rotating; empty bars: counter rotating modes) [12].

\section{Feasibility study taking into account the field profile of the new $10.5 \mathrm{~T}$ magnet}

The theoretical feasibility of operation at higher frequencies was shown in the previous section. Since the frequency of operation is proportional to the magnetic field, the magnetic field has to be increased for higher frequencies (e.g. $204 \mathrm{GHz}$ leads to $\sim 8.15 \mathrm{~T}$ and $238 \mathrm{GHz}$ leads to $\sim 9.45 \mathrm{~T}$ ). The current test stand at KIT is limited to the maximum magnetic field strength of $\sim 6.9 \mathrm{~T}$ of the existing Oxford Instruments magnet. Therefore, KIT ordered a new SC magnet including the following key features:

- Maximum field at cavity: $10.5 \mathrm{~T}$,

- Warm bore hole diameter: $261 \mathrm{~mm}$,

- Dipole coils for electron beam alignment,

- Cryogen-free cooling system.

The first question to be answered is to assess whether the currently operating coaxial gyrotron can also operate in the new magnet without any modifications. Therefore, the magnetic field configuration from Tesla has been used for interaction simulations. The simulated adiabatic start-up scenario, assuming a kinetic energy spread of $\Delta E=0.2 \% \mathrm{rms}$ and a relatively large spread of $\Delta \alpha=20 \% \mathrm{rms}$ for the electron velocity ratio $\alpha$, shown in Fig. 3 has a smooth start up where only co-rotating modes have been excited.

In a second step, the new coaxial MIG with nonemissive coating of the emitter edges [15], delivered by Thales Electron Devices, is considered. The electron beam parameters have been obtained using ARIADNE $[14,16]$. The simulations show a huge number of trapped electrons and/or high alpha spread of around $\Delta \alpha=40 \%$ rms at the maximal velocity ratio of $\alpha=1.12$, due to the different length of the new magnet and, therefore, misplacement of the gun. For this reason, the distance between emitter and cavity has to be adjusted to the field profile of the new Tesla magnet. This issue can be solved by e.g. inserting a spacer into the beam tunnel. Further, the coaxial insert has to be redesigned, to keep

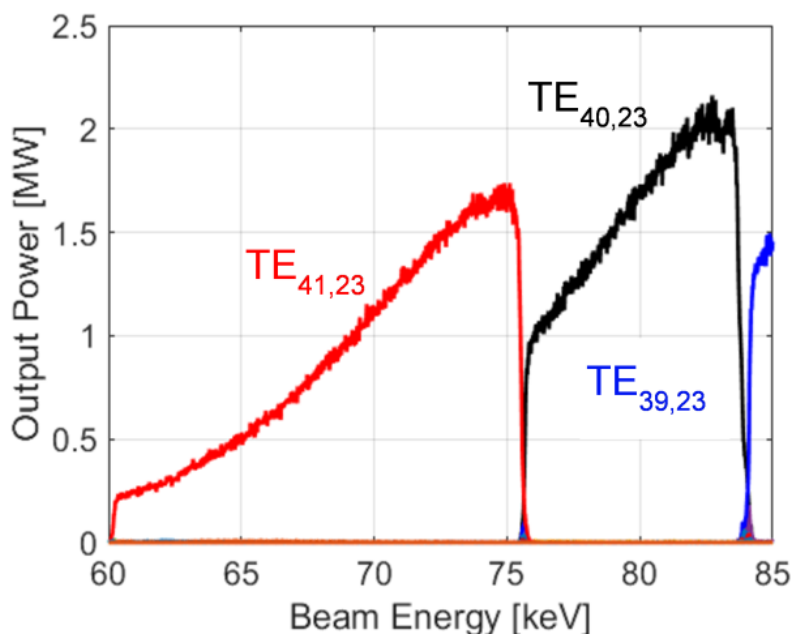

Fig. 2. Adiabatic start-up scenario with existing coaxial cavity at $8.15 \mathrm{~T}$ and $I_{\text {beam }}=70 \mathrm{~A}$ under the consideration of the magnetic field profile of the Oxford Instruments magnet. 
the insert corrugations to the right place in the cavity.

This adjustment has been taken into account in the following simulations shown in this paper.

\section{More Realistic Start-Up Scenario}

After checking the existing setup of the pre-prototype, simulations using the longer beam tunnel have been performed. Realistic multi-mode start-up simulations have been made considering the coaxial cavity with the expected magnetic field profile of the new magnet and the corresponding beam parameters using the coaxial MIG with non-emissive coating of the emitter edges at $170 \mathrm{GHz}\left(\mathrm{TE}_{34,19}\right.$ mode) and $204 \mathrm{GHz}$ ( $\mathrm{TE}_{40,23}$ mode). The more realistic scenario considers also the nonadiabaticity of electron trajectory close to the emitter Further, the simulation results with and without nonlinear uptaper have been compared to each other. Additionally, a modification of the cavity has been taken into account for an improved performance.

\subsection{Simulation results at $170 \mathrm{GHz}$ using the existing coaxial-cavity design}

Firstly, ARIADNE simulations using the new magnetic field profile have been performed and the obtained electron beam parameters have been used in EURIDICE. In this simulation, at $170 \mathrm{GHz}$ the alpha spread is $\Delta \alpha=6.08 \%$ for $\alpha=1.3$. The energy spread amounts to $0.08 \%$ and the kinetic energy spread to $0.12 \%$. The realistic start-up scenario at $6.86 \mathrm{~T}$ without non-linear uptaper is shown in Fig. 4. Taking the wall loading constraint into account, the operation parameters are $U_{\text {beam }}=89 \mathrm{keV}$ and $I_{\text {beam }}=75 \mathrm{~A}$, where the tube can deliver an RF output power of $2.3 \mathrm{MW}$ at an interaction efficiency of $36.7 \%$. Initial studies including the nonlinear uptaper after the cavity have been started. Figure 5 presents the start-up scenario using the same parameters as mentioned before for a better comparison. The shown start-up is not that smooth as before, but nevertheless the nominal $\mathrm{TE}_{34,19}$ mode is finally excited. The wall loading constraint limits the operation to $U_{\text {beam }}=90 \mathrm{keV}$. The RF

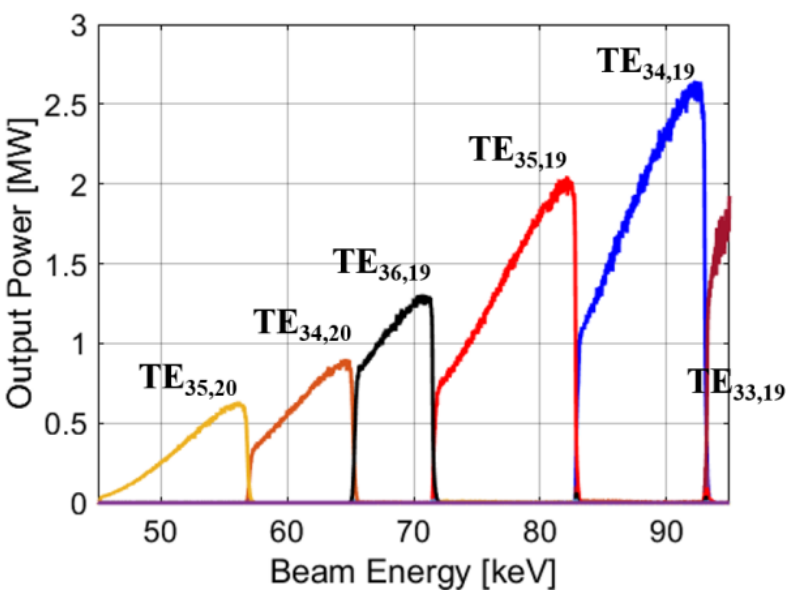

Fig. 3. Realistic start-up scenario with existing coaxial cavity at $6.86 \mathrm{~T}$ and $I_{\text {beam }}=75 \mathrm{~A}$ without non-linear uptaper using the magnetic field profile proposal from the new SC magnet and the gun parameters from the new coaxial MIG. output power decreases to $2.25 \mathrm{MW}$ at an interaction efficiency of $34 \%$ and an insert loading of $0.12 \mathrm{~kW} / \mathrm{cm}^{2}$.

\subsection{Simulation results at $204 \mathrm{GHz}$ using the existing coaxial-cavity design}

The coaxial MIG with non-emissive coating of the emitter edge have been simulated via ARIADNE for the operation at $204 \mathrm{GHz}$ using the new magnet. The gun parameters have been selected to give a pitch factor of $\alpha=1.2$ having an alpha spread of $\Delta \alpha=4.3 \%$ and a kinetic energy spread of $0.1 \%$. The simulation shows a good scenario starting with the TE $-40,24$ mode at $\sim 48 \mathrm{keV}$ in Fig. 6, where the minus refers to a counter-rotating mode. Starting from $60 \mathrm{keV}$ the $\mathrm{TE}_{41,23}$ mode is excited while the nominal $\mathrm{TE}_{40,23}$ mode is getting dominant at $\sim 75 \mathrm{keV}$. Starting from $83 \mathrm{keV}$ the competing TE-38,23 mode, which has nearly the same caustic radius, is getting stronger and so the nominal mode collapses. The operation is limited to $79.2 \mathrm{keV}$ due to the wall loading constraint. The cavity delivers $1.7 \mathrm{MW}$ RF output power without non-linear uptaper at an interaction efficiency of

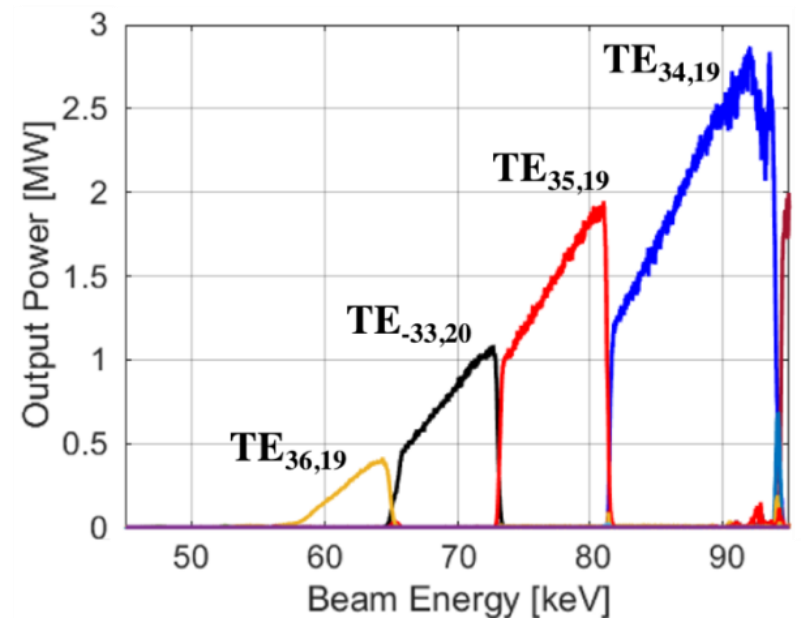

Fig. 4. Realistic start-up scenario with the existing coaxial cavity at $6.86 \mathrm{~T}$ and $I_{\text {beam }}=75 \mathrm{~A}$ without non-linear uptaper using the magnetic field profile proposal from the new SC magnet and the gun parameters from the new coaxial MIG.

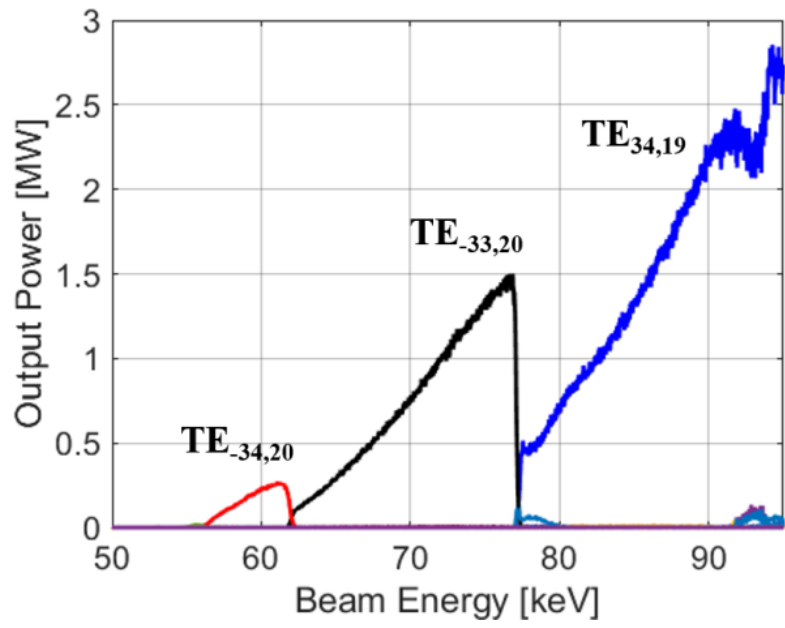

Fig. 5. Realistic start-up scenario with existing coaxial cavity at $6.86 \mathrm{~T}$ and $I_{\text {beam }}=75 \mathrm{~A}$ with non-linear uptaper using the magnetic field profile proposal from the new SC magnet and the gun parameters from the new coaxial MIG. 
$33 \%$ and an insert loading of $0.06 \mathrm{~kW} / \mathrm{cm}^{2}$. The delivered RF output power is $0.1 \mathrm{MW}$ lower than the results presented in section 3 .

Initial studies considering also the influence of the non-linear uptaper at $204 \mathrm{GHz}$ are being performed. Figure 7 presents the start-up scenario using the same parameters as mentioned before for comparing the results to each other. The scenario looks quite similar with and without the non-linear uptaper, as it should. The point at which the $\mathrm{TE}_{40,23}$-mode collapses is nearly identical as before. Additionally, the $\mathrm{TE}_{41,22}$ mode oscillates after the $\mathrm{TE}_{40,22}$ mode. The delivered $\mathrm{RF}$ output power and the interaction efficiency are not influenced by the non-linear uptaper.

\subsection{Further modifications}

In order to get a dual-frequency gyrotron delivering $2 \mathrm{MW}$, or even more at both operation frequencies, modifications have to be done. For this reason, the cavity midsection length has been reduced from $16 \mathrm{~mm}$ to $13.6 \mathrm{~mm}$, as mentioned in the previous section. The start-up simulation of the shortened cavity for the $\mathrm{TE}_{34,19}$-mode case is shown in Fig. 8. Concerning the nominal operation parameters of $U_{\text {beam }}=90 \mathrm{keV}$ and $I_{\text {beam }}=75 \mathrm{~A}$ and an increased magnetic field of $6.88 \mathrm{~T}$, the cavity delivers $2.4 \mathrm{MW}$ RF output power at an interaction efficiency of $37 \%$ having a wall loading of $1.9 \mathrm{~kW} / \mathrm{cm}^{2}$. Compared with the longer cavity, the gyrotron can deliver $0.1 \mathrm{MW}$ more RF output power with an increased interaction efficiency of $\sim 1$ percentage point at this operating point. However, concerning the Ohmic wall loading constraint the nominal operation point could be extended to $U_{\text {beam }}=90.6 \mathrm{keV}$, where the cavity delivers $2.5 \mathrm{MW}$ at an efficiency of $38.2 \%$.

This modification has to be proven for the $204 \mathrm{GHz}$ operation, as well. The simulation shows a good scenario starting with the $\mathrm{TE}_{42,23}$ mode, over the $\mathrm{TE}_{41,23}$ mode and finally the nominal $\mathrm{TE}_{40,23}$ mode is excited until the competing TE-38,23 mode raises, as shown in Fig. 9. The operation point is found by the wall loading constraint and is determined by $U_{\text {beam }}=86.5 \mathrm{keV}$, having an RF

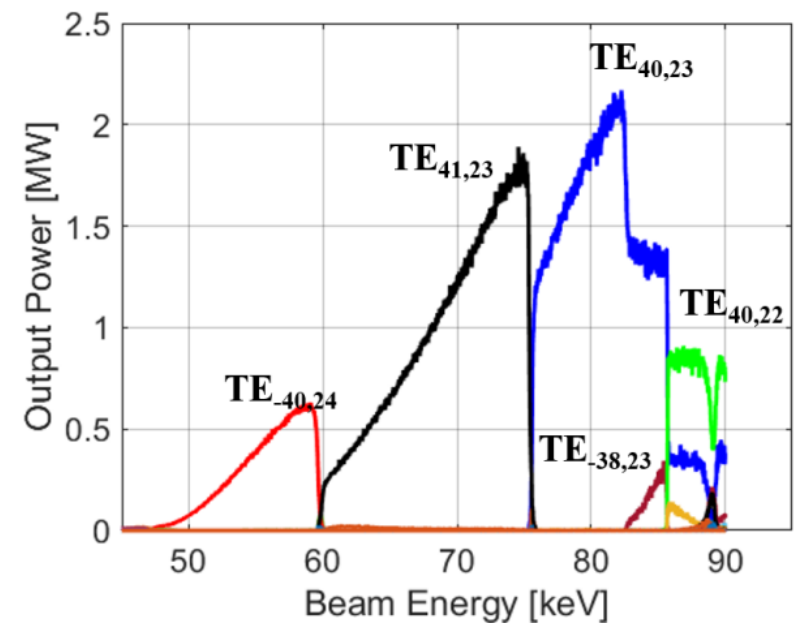

Fig. 6. Realistic start-up scenario with the existing coaxial cavity at $8.15 \mathrm{~T}$ and $I_{\text {beam }}=70 \mathrm{~A}$ without non-linear uptaper using the magnetic field profile proposal from the new SC magnet and the gun parameters from the new coaxial MIG. output power of $2.06 \mathrm{MW}$ at an interaction efficiency of $33.5 \%$. Comparing these results with the existing cavity design, the RF output power can be increased by $\sim 0.4 \mathrm{MW}$ and the interaction efficiency by 0.5 percentage points.

\section{Multi-Frequency Launcher Design}

The operating modes excited in the cavity are converted into the fundamental Gaussian mode using an internal quasi-optical mode converter which contains a mirrorline launcher and three mirrors. The existing single frequency $(170 \mathrm{GHz})$ launcher design is already presented in [10]. The simulation results show that in the case of fabrication error on the wall perturbation of \pm $10 \mu \mathrm{m}$, the launcher will still provide a RF beam with high Gaussian mode content. The measurement results are in a quit good agreement with the simulations. The simulated fundamental Gaussian mode content is determined by $96.3 \%$ at the launcher aperture at $170 \mathrm{GHz}$ [17].

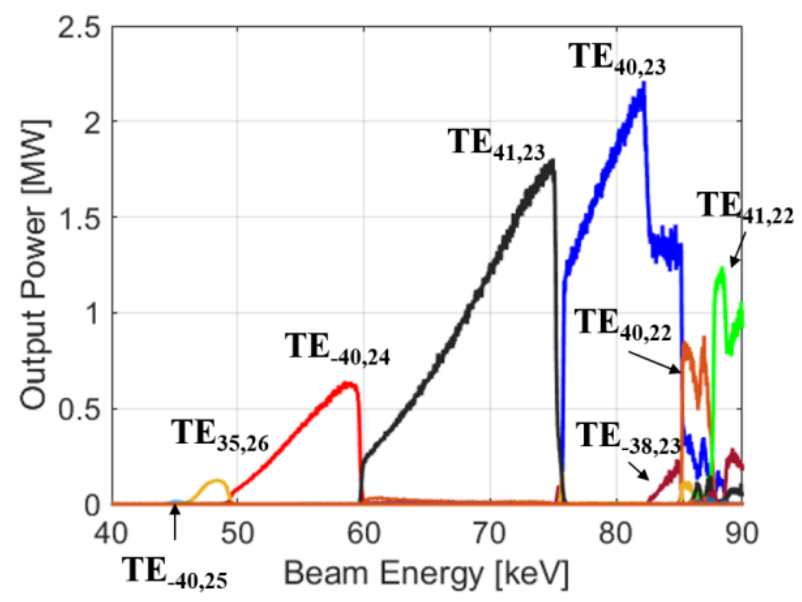

Fig. 7. Realistic start-up scenario with the existing coaxial cavity at $8.15 \mathrm{~T}$ and $I_{\text {beam }}=70 \mathrm{~A}$ including the non-linear uptaper using the magnetic field profile proposal from the new $\mathrm{SC}$ magnet and the gun parameters from the new coaxial MIG.

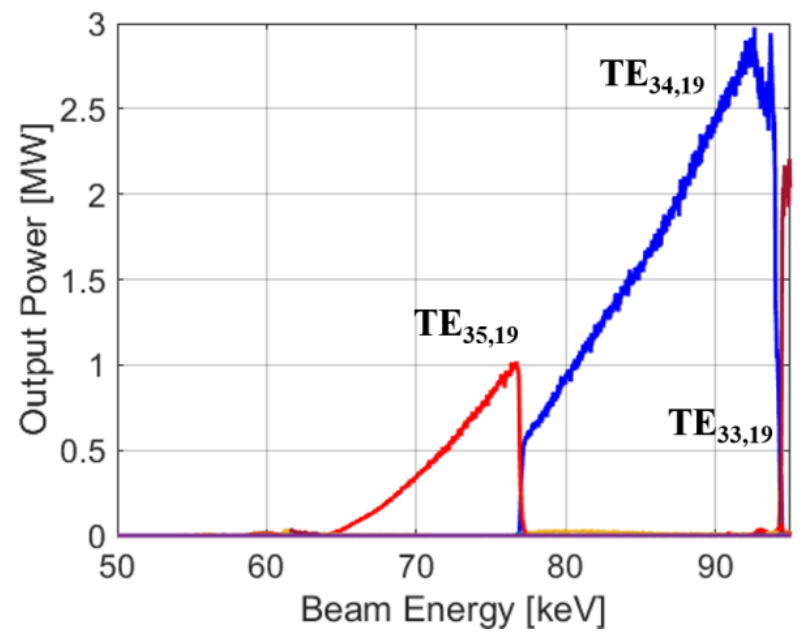

Fig. 8. Realistic start-up scenario with a shortened coaxial cavity length at $6.88 \mathrm{~T}$ and $I_{\text {beam }}=75 \mathrm{~A}$ using the magnetic field profile proposal from the new SC magnet and the gun parameters from the new coaxial MIG. 
Table 2. Summary of the realistic multi-mode simulation results using EURIDICE using the new Tesla magnet configuration.

\begin{tabular}{|l|c|c|c|c|c|c|}
\hline Mode & \multicolumn{3}{|c|}{$\mathrm{TE}_{34,19}$} & \multicolumn{3}{c|}{$\mathrm{TE}_{40,23}$} \\
\hline Configuration & W.o. uptaper & W. uptaper & modification & W.o. uptaper & W. uptaper & modification \\
\hline Frequency [GHz] & 170.005 & 170.020 & 170.041 & 204.145 & 204.141 & 204.167 \\
\hline Beam voltage [keV] & 90 & 90 & 90.6 & 79.2 & 79.2 & 86.5 \\
\hline Beam current [A] & 75 & 75 & 75 & 70 & 70 & 75 \\
\hline Magnetic field [T] & 6.86 & 6.86 & 6.88 & 8.15 & 8.15 & 8.23 \\
\hline Velocity ratio, $\alpha$ & 1.3 & 1.3 & 1.3 & 1.2 & 1.2 & 1.2 \\
\hline Interaction length [mm] & 16.0 & 16.0 & 13.6 & 16.0 & 16.0 & 13.6 \\
\hline Wall loading [kW/cm $\left.{ }^{2}\right]$ & 1.89 & 2.00 & 2.00 & 2.00 & 2.00 & 2.00 \\
\hline Insert loading [kW/cm ${ }^{2}$ ] & 0.11 & 0.11 & 0.13 & 0.06 & 0.06 & 0.07 \\
\hline RF output power [MW] & 2.3 & 2.2 & 2.5 & 1.7 & 1.7 & 2.06 \\
\hline Interaction eff. [\%] & 36.7 & 34.0 & 38.2 & 33 & 33 & 33.5 \\
\hline
\end{tabular}

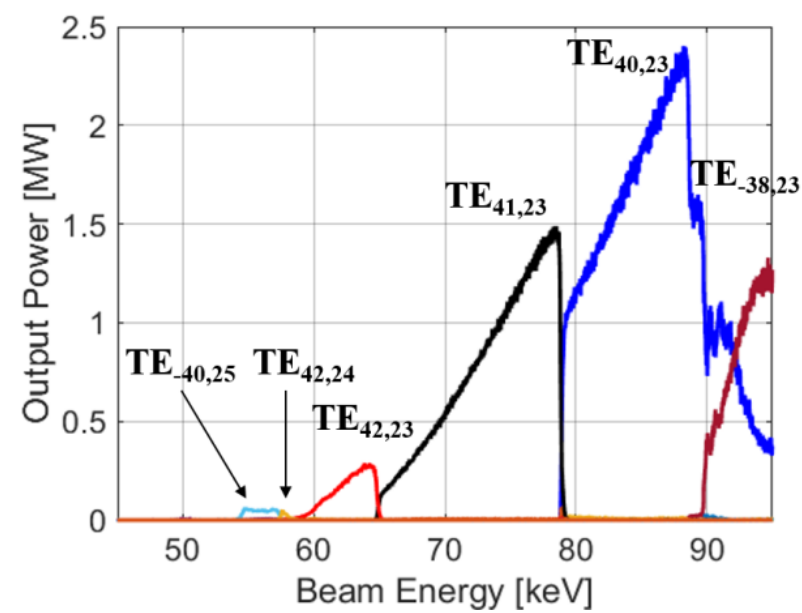

Fig. 9. Realistic start-up scenario with a shortened coaxial cavity midsection length at $8.23 \mathrm{~T}$ and $I_{\text {beam }}=75 \mathrm{~A}$.

Having a dual frequency scenario, the existing mirrorline launcher with radius of $32.5 \mathrm{~mm}$ is tested to assess whether the performance is good enough at $204 \mathrm{GHz}$. First initial studies at $204 \mathrm{GHz}$ for the $\mathrm{TE}_{40,23}$ mode show a fundamental Gaussian mode content of only $91.6 \%$ at the launcher aperture. Therefore, a new launcher was designed with good performance operating both at $170 \mathrm{GHz}$ and $204 \mathrm{GHz}$. The new launcher is also a mirror-line launcher, and its radius is $32 \mathrm{~mm}$. This dual-frequency launcher design shows promising results despite the difference of the modes' caustic radii of around $2 \%$. The Gaussian mode content was calculated to be $97.2 \%$ at $170 \mathrm{GHz}$ and $96.6 \%$ at $204 \mathrm{GHz}$.

\section{Summary and Outlook}

The simulations of this paper show that the existing KIT $\mathrm{TE}_{34,19}$-mode coaxial-cavity pre-prototype gyrotron could operate in the KIT FULGOR gyrotron test stand using the new 10.5 T magnet, under the assumption of enlarge the beam tunnel. Furthermore, the adapted gyrotron is capable of operation as a multipurpose/multi-frequency device. Without any other modifications the simulations show that the gyrotron delivers at $204 \mathrm{GHz}(170 \mathrm{GHz}) 1.7 \mathrm{MW}(2.3 \mathrm{MW})$ at an interaction efficiency of $33 \%(36.7 \%)$. A small modification of the cavity length would increase the RF output power by $0.4 \mathrm{MW}$ and the interaction efficiency by 0.5 percentage points at $204 \mathrm{GHz}$. Operating a dual- frequency coaxial-cavity gyrotron in the new test stand, the following aspects have to be taken into account: (i) the gyrotron length has to be adjusted due to the height of the new magnet, (ii) as a consequence to this, the coaxial insert has to be modified by the same length, (iii) in principle the cavity can operate at both frequencies, but with a small reduction of the cavity length the results can be improved, and (iv) the launcher has to be improved for sufficient performance at $204 \mathrm{GHz}$.

This work has been carried out within the framework of the EUROfusion Consortium and has received funding from the Euratom research and training programme 2014-2018 under grant agreement No. 633053. The views and opinions expressed herein do not necessarily reflect those of the European Commission. Part of the simulations were performed on the EUROfusion High Performance Computer (MarconiFusion).

\section{References}

1. H. Braune, et al., In Proc. IRMMW (2016).

2. T. Omori, et al., Fusion Eng. Des., (2011).

3. G. Federici, et al., in Proc. 25 $5^{\text {th }}$ SOFE, (2013).

4. Z. C. Ioannidis et al., IEEE Trans. on Electron Devices, (2017).

5. P. C. Kalaria, et al., Physics of Plasma, 23(9), 2016.

6. C. T. Iatrou, et al., IEEE Trans. Microwave Theory Tech., (1996).

7. K. A. Avramides, et al., "IEEE Trans. on Plasma Science, (2004).

8. T. Rzesnicki, et al., "IEEE Trans. on Plasma Science, (2010).

9. S. Illy, et al., EC-20 workshop, (2018).

10. S. Ruess, et al. , $47^{\text {th }}$ EUMC, (2017).

11. M. Schmid et al., Fus. Eng. Des. 123, 485, 2017.

12. T. Ruess, et al., GeMiC (2018).

13. K. A. Avramides, et al., EC-17 Workshop (2012).

14. K. A. Avramidis et al., IRMMW-THz, Copenhagen, 2016, pp. 1-2.

15. I. Gr. Pagonakis, S. Illy, M. Thumm, Physics of Plasmas, 23, 83103 (2016).

16. I. Gr. Pagonakis and J. L. Vomvoridis. IRMMW (2004).

17. J. Jin, et al., in IEEE Trans. on Microwave Theory and Techniques (2009). 